\title{
Patrick Vallance
}

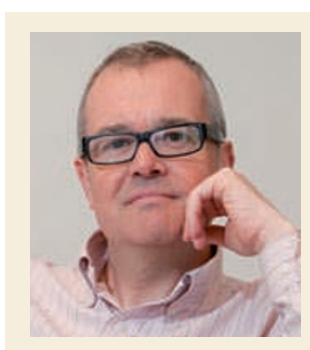

Senior Vice President of Medicines Discovery and Development, GlaxoSmithKline, UK. Prior to joining GlaxoSmithKline (GSK) in May 2006, Patrick Vallance was a clinical academic and led the Division of Medicine at University College London, UK. He has more than 20 years of experience in clinical medicine and was elected to the Academy of Medical Sciences in 1999. He was Registrar of the Academy of Medical Sciences until he moved to GSK. Now, Vallance is responsible for GSK's pharmaceutical pipeline from the initial chemical hit against a target through to the completion of a medicine's development. $\mathrm{He}$ is also an advocate for greater interactions between the pharmaceutical industry, academic institutions and biotech to help stimulate innovation.

What have been the main successes and failures since GSK created the Centre of Excellence for Drug Discovery (CEDD) model for research and development (R\&D) in 2006? The basic CEDD premise of bringing together the various specialities that are important for drug discovery — such as biology, chemistry and clinical research - into one integrated unit is exactly right. You do not want them to be separated into decentralized functions. However, we learnt a lot of lessons from the original CEDDs that we have tried to apply downstream. The first is that we now know that the scale of the CEDDs was not exactly right as some of them were quite large; that is, 250-400 people. So, we formed Discovery Performance Units (DPUs), which include 50-60 people. The second lesson learnt was that we found co-locating our DPU teams to be essential for getting a biotech-like spirit for day-to-day interactions. And the third lesson learnt is focus. Some of the CEDDs covered two or three different therapeutic areas and as a result there were winners and losers. The DPUs allow us to have an absolutely relentless focus on one area.

Overall, the CEDD model allowed us to apply an integrated approach to take a hypothesis very quickly into the clinic without having big boundaries when moving a drug from the preclinical to clinical stage. This has allowed very rapid progression of innovative molecules through the pipeline. The lessons learnt from the CEDDs allowed us to iterate that back down to a more refined version to create the DPUs. One advantage of the DPU model is the individual scientist's opportunity to become an integrated drug discoverer. Usually such accountability only occurs at senior levels, but in a DPU, like at a biotech company, that accountability is at a more junior level. Therefore, you start to have a whole raft of integrated drug discoverers, which is an important skill in industry. The downside is that some molecules do not make it, or you may end up in a research area that is not going anywhere. So, there will be failures, but in a DPU the failures are visible and you can try to understand them. One of the dangers in very large organizations is that you cannot see failure or success happening. Small integrated accountable teams are also the focus of our late-stage organization the so-called medicines development teams.

How is GSK building the need to demonstrate value to reimbursement agencies into the early drug R\&D process?

This is a really interesting area for me because I come from a background of 20 years as a clinician and as an academic, and I have sat on reimbursement panels. In more recent years, the fundamental switch is that the payers have started to define innovation in terms of what they are prepared to pay for, rather than industry defining innovation in terms of what they are prepared to give.

So, we have a whole process in place starting with strategy documents, called medicines vision documents, that start to define what the differences are in any particular medicine, target or approach that we think everyone would recognize as being different enough to warrant paying for from a fixed budget. We have more work to do to get this properly embedded into the early stages of the organization and to ensure more integration of early and late drug development, but we are definitely on track. I also want groups to use role play to behave like a mock reimbursement panel as I think that will help them to get into the mindset of how a panel would behave with a fixed pot of money to spend on health care.

In your view, what are the main challenges of developing drugs for rare diseases and, now that GSK has a dedicated unit specializing in this area, how can the company help to address them?

Approximately 7,000 rare diseases have been identified worldwide. But, if you look across at all of the major pharmaceutical companies, we have medicines for only $80-90$. So, there is a massive unmet need that we aim to help address. Also, for some rare diseases particularly those of genetic origin - you remove some of the uncertainties that you have when you are developing treatments for common diseases. For example, you know the cause and effect of the disease, you know how to diagnose patients and you therefore know how to recruit the right patients. From a drug development perspective, you can do a more focused discovery programme with a greater idea of the validity of your target early on.

I also think that studying rare diseases begins to foreshadow how common diseases might eventually fragment. Currently, many of the disease labels we have are nineteenth or twentieth century definitions of diseases. They are not molecular or cellular classifications of subtypes. So, as we fragment common diseases, and we are already seeing this in oncology, they start to look a bit more like rare diseases. It may be a long time before we get there, but that is the direction in which we seem to be going.

The challenges are that it is sometimes difficult to find patients as they are often scattered around the world and, although it should be easy to diagnose them, some patients remain undiagnosed because they do not have access to a regional specialist or they are misdiagnosed. Of course, some of the rare diseases are as poorly understood as some of the common diseases and those are going to be really difficult to develop medicines for because then you have got all of the complexity of common disease drug discovery with the added complexity of rarity and difficulty in understanding how you are going to get the product to the market. So, you have to be careful in choosing the rare diseases that you think are tractable. 